\title{
Exponential stabilization of stochastic quantum systems via combined feedback control
}

\author{
Jie Wen, Yuanhao Shi, Jianfang Jia and Jianchao Zeng
}

\begin{abstract}
We propose a novel control strategy by combining state feedback and noise-assisted feedback to exponentially stabilize the target eigenstate for two-level stochastic quantum systems in this paper. The state space is divided into two subspaces, and the state feedback and noise-assisted feedback work in the corresponding subspace, respectively, to achieve the faster state convergence than that of using the two feedback strategies individually. Two kinds of continuous noise-assisted feedback controls are used to form the combined feedback strategies, respectively, and the exponential stabilization of target eigenstate is proved. The effectiveness and superiority of the combined feedback strategies are also verified in numerical simulations.
\end{abstract}

Index Terms-exponential stabilization, stochastic quantum systems, state feedback, noise-assisted feedback.

\section{INTRODUCTION}

$\mathbf{T}$ HE preparation of eigenstates is one of the basic tasks in quantum technologies, such as quantum computing, quantum communication and quantum measurement. Since the early 1980s, many classical control methods, e.g. optimal control [1, 2], sliding mode control [3, 4], robust control [5], and Lyapunov control [6-8], have be introduced and applied in quantum systems to prepare eigenstate accurately and rapidly.

In order to obtain better robustness, closed-loop feedback control is considered for quantum systems. Unfortunately, the system information are needed in closed-loop feedback control, and the measurement can lead to the collapse of quantum states. To address this problem, Belavkin proposed the quantum filter theory $[9,10]$, whose a modern introduction can be found in [11]. Based on quantum filter theory, the measurement-based feedback control is developed, in which the measured quantum state is used as the input of quantum filter while the state of quantum filter is used in feedback control.

Under continuous measurement, the state evolution of quantum filter can be describe by a Ito stochastic differential equation, known as quantum stochastic master equation (SME) [12-14]. Based on SME, the global stabilization of target eigenstate for quantum spin systems [12], $N$-level quantum angular moment systems $[13,15,16]$ and the general

This work was supported in part by National Natural Science Foundation of China under Grant 61803350, in part by the Natural Science Foundation of Shanxi Province under Grant 201801D221208 and Grant 201801D121159, and in part by Scientific and Technological Innovation Programs of Higher Education Institutions in Shanxi under Grant 2019L0583.

J. Wen, Y. Shi and J. Jia are with the School of Electrical and Control Engineering, North University of China, Taiyuan 030051, China (e-mail: wenjie015@gmail.com; yhshi@nuc.edu.cn;jiajianfang@nuc.edu.cn).

J. Zeng is with School of Data Science and Technology, North University of China, Taiyuan 030051, China (email: zengjianchao@263.net). finite-dimensional stochastic quantum systems [17], had been achieved by using switching control [13], continuous control $[15,16]$ and non-smooth state feedback control [17], respectively, to overcome the symmetric structure of state space. Further, Vu et al. proposed the coupling Lyapunov functions approach to obtain faster state convergence by reducing the switching times of switching control [18]. In particular, Liang et al. designed a continuous state feedback control to achieve the exponential stabilization of eigenstate for spin- $\frac{1}{2}$ systems [19] and $N$-level quantum angular momentum systems [20] based on the Support theorem [21] and stochastic LaSalletype theorem [22], and the robustness when the initial state and physical parameters are unknown was also investigated in [23, 24], while Cardona et al. proposed the noise-associated feedback strategy to exponentially stabilize eigenstate for $N$ level quantum systems [25] based on the exponentially stable theory of stochastic differential equations [26]. Recently, we developed the noise-associated feedback strategy by using continuous functions instead of the saturating function used in [25] as the noise gain functions in [27].

According to the literature review, both of state feedback [19, 20] and noise-assisted feedback [25, 27] can achieve the exponential stabilization of eigenstate. However, in noiseassisted feedback strategy, the noise helps to shake the system state away from the bad equilibra but also hinders the attraction of target state, while the state convergence under state feedback is slower than that under noise-assisted feedback. In order to further improve the state convergence rate, we combine state feedback and noise-assisted feedback to exponentially stabilize the target eigenstate by dividing the state space into two subspace and using the two feedback controls in the corresponding subspace, respectively. This control strategy is denoted as combined feedback control in this paper. We will present the design of combined feedback control, and prove the exponential convergence as well as the superiority of combined feedback, which are also verified in numerical simulations.

The rest of this paper is organized as follows. The model of two-level quantum system under the combined feedback and the exponential convergence problem of eigenstates are described in Section II. We propose the combined feedback strategies, and prove the exponential convergence in Section III, in which we also compare the combined feedback with state feedback and noise-assisted feedback. In Section IV, numerical simulations are performed to verify the effectiveness and superiority of the combined feedback. Finally, we conclude this paper and briefly discuss possible future directions in Section V.

Notation. $\mathbb{R}$ denotes the set of real numbers. $\mathbb{C}$ denotes the 
set of complex numbers. $\mathbb{C}^{m \times n}$ denotes the set of complexentried $m \times n$ matrices. $A^{\dagger}$ is the conjugate transpose of the matrix $A . \operatorname{tr}(A)$ denotes the trace of matrix $A . \sigma_{x}=$ $\left[\begin{array}{ll}0 & 1 \\ 1 & 0\end{array}\right], \sigma_{y}=\left[\begin{array}{cc}0 & -\mathrm{i} \\ \mathrm{i} & 0\end{array}\right]$ and $\sigma_{\mathrm{z}}=\left[\begin{array}{cc}1 & 0 \\ 0 & -1\end{array}\right]$ denote the Pauli matrices, where $\mathrm{i}=\sqrt{-1} \cdot[A, B]=A B-B A$ denotes the commutator of matrices $\mathrm{A}$ and $\mathrm{B}$. $\mathcal{L}$ denotes the infinitesimal generator. $\mathbb{E}[X]$ denotes the expected value of a random variable $X$.

\section{PROBLEM FORMULATION}

The state evolution of two-level quantum systems under continuous measurement can be described by the following Ito stochastic differential equations

$$
\begin{aligned}
d \rho_{t}= & \left(L \rho_{t} L^{\dagger}-\frac{1}{2}\left(L^{\dagger} L \rho_{t}+\rho_{t} L^{\dagger} L\right)\right) d t \\
& +\sqrt{\eta}\left(L \rho_{t}+\rho_{t} L^{\dagger}-\operatorname{tr}\left(\rho_{t}\left(L+L^{\dagger}\right)\right) \rho_{t}\right) d W_{t}
\end{aligned}
$$

where, $\rho_{t}$ is the density matrix of describing quantum state, and evolves in the state space $S$, i.e. $\rho_{t} \in S=$ $\left\{\rho_{t} \in \mathbb{C}^{2 \times 2}: \rho_{t}=\rho_{t}^{\dagger} \geq 0, \operatorname{tr}\left(\rho_{t}\right)=1, \operatorname{tr}\left(\rho_{t}^{2}\right) \leq 1\right\} ; \quad L$ is the measurement operator; $W_{t}$ is a standard Wiener process defined on the classical complete probability; $\eta$ is the measurement efficiency. The equation (1) is known as quantum stochastic master equation (SME), and the existence and uniqueness of the solution of quantum SME have been proved in $[13,28]$.

In this paper, we address the problem of exponential stabilization of eigenstate for the quantum systems (1), i.e., the system state can exponentially converge to the target eigenstate from arbitrary initial state in the state apace $S$. In order to guarantee the problem solvability, the target eigenstate should be one of the equilibria of SME (1), so we consider the following assumption made on the system (1).

Assumption 1. The measurement operator $L$ is self-adjoint and regular, i.e., $L=L^{\dagger}$ and the eigenvalues of $L$ are different.

Assumption 1 is practically reasonable as in many physical experiment settings, e.g., trapping a cold atomic ensemble in an optical cavity $[12,29,30]$. Based on Assumption 1, the equilibria of SME (1) are all the eigenstates of measurement operator $L$ [17], and SME (1) can be written as

$$
\begin{aligned}
d \rho_{t}= & \left(L \rho_{t} L-\frac{1}{2}\left(L^{2} \rho_{t}+\rho_{t} L^{2}\right)\right) d t \\
& +\sqrt{\eta}\left(L \rho_{t}+\rho_{t} L-2 \operatorname{tr}\left(\rho_{t} L\right) \rho_{t}\right) d W_{t}
\end{aligned}
$$

However, the system state converges to an arbitrary equilibrium with a certain probability without the control input [19]. In order to make sure that the system state converges to the target eigenstate instead of arbitrary eigenstate, the suitable controllers need to be used. For this goal, lots of control strategies have been proposed, e.g. static output feedback [31-33], state feedback [12-14, 19, 20, 34, 35] and noiseassisted feedback [25, 27]. In particular, both of the state feedback in [19] and the noise-assisted feedback in [27] can achieve the exponential stabilization of eigenstate for two-level stochastic quantum systems. The state feedback and noiseassisted feedback take the form

$$
u_{t}^{s f} d t=f\left(\rho_{t}\right) d t
$$

and

$$
u_{t}^{n f} d t=g\left(\rho_{t}\right) d B_{t}
$$

respectively, where $B_{t}$ is an exogenous Brownian noise independent of $W_{t}$.

In order to make the best of the advantages of state feedback and noise-assisted feedback, we propose the combined feedback strategy by combining the two feedback, i.e.,

$$
\begin{aligned}
u_{t}^{c f} d t & =\kappa\left(\rho_{t}\right) u_{t}^{s f} d t+\left(1-\kappa\left(\rho_{t}\right)\right) u_{t}^{n f} d t \\
& =\kappa\left(\rho_{t}\right) f\left(\rho_{t}\right) d t+\left(1-\kappa\left(\rho_{t}\right)\right) g\left(\rho_{t}\right) d B_{t}
\end{aligned}
$$

where, $\kappa\left(\rho_{t}\right) \in\{0,1\}$. Under the combined feedback 5, (2) becomes

$$
\begin{aligned}
d \rho_{t}= & \left(L \rho_{t} L-\frac{1}{2}\left(L^{2} \rho_{t}+\rho_{t} L^{2}\right)\right) d t-\mathrm{i} \kappa\left(\rho_{t}\right) f\left(\rho_{t}\right)\left[H, \rho_{t}\right] d t \\
& +\left(1-\kappa\left(\rho_{t}\right)\right)^{2} g(\rho)^{2}\left(H \rho_{t} H^{\dagger}-\frac{1}{2}\left(H^{\dagger} H \rho_{t}+\rho_{t} H^{\dagger} H\right)\right) d t \\
& +\sqrt{\eta}\left(L \rho_{t}+\rho_{t} L-2 \operatorname{tr}\left(\rho_{t} L\right) \rho_{t}\right) d W_{t} \\
& -\mathrm{i}\left(1-\kappa\left(\rho_{t}\right)\right) g\left(\rho_{t}\right)\left[H, \rho_{t}\right] d B_{t}
\end{aligned}
$$

where, $H=H^{\dagger}$ is the actuator Hamiltonian from $\rho_{t+d t}=$ $e^{-\mathrm{i} H u_{t} d t}\left(\rho_{t}+d \rho_{t}\right) e^{\mathrm{i} H u_{t} d t}$ [25]. Let $L=\sqrt{\Gamma} \sigma_{z}$ and $H=\sigma_{y}$, where $\Gamma$ is the measurement strengths, (6) can be written as

$$
\begin{aligned}
d \rho_{t}= & \Gamma\left(\sigma_{z} \rho_{t} \sigma_{z}-\rho_{t}\right) d t-\mathrm{i} \kappa\left(\rho_{t}\right) f\left(\rho_{t}\right)\left[\sigma_{y}, \rho_{t}\right] d t \\
& +\left(1-\kappa\left(\rho_{t}\right)\right)^{2} g\left(\rho_{t}\right)^{2}\left(\sigma_{y} \rho_{t} \sigma_{y}-\rho_{t}\right) d t \\
& +\sqrt{\eta \Gamma}\left(\sigma_{z} \rho_{t}+\rho_{t} \sigma_{z}-2 \operatorname{tr}\left(\rho_{t} \sigma_{z}\right) \rho_{t}\right) d W_{t} \\
& -\mathrm{i}\left(1-\kappa\left(\rho_{t}\right)\right) g\left(\rho_{t}\right)\left[\sigma_{y}, \rho_{t}\right] d B_{t}
\end{aligned}
$$

which is the controlled two-level quantum system considered in this paper.

The idea of the combined feedback strategy is that the state space is divided into two subspaces, and the state convergence under state feedback is faster than that under noise-assisted feedback when the state is located in one subspace while the state convergence under noise-assisted feedback is faster when the state is located in the other subspace, so that the state convergence under the combined feedback is faster than that under state feedback and noise-assisted feedback individually for the entire state space. Thus, the key of designing the combined feedback strategy is to determine how to divide the state space, which is used to obtain $\kappa\left(\rho_{t}\right)$, and the goal of this paper can be described as follows.

Exponential stabilization by combined feedback: for the two-level quantum SME (7), design the combined feedback control $u_{t}^{c f} d t=\kappa\left(\rho_{t}\right) u_{t}^{s f} d t+\left(1-\kappa\left(\rho_{t}\right)\right) u_{t}^{n f} d B_{t}$, to exponentially render the SME (7) from any initial state $\rho_{0}$ in the state space $S$ to the desired target eigenstate $\rho_{f}$, and make sure that the state exponential convergence is faster than that of using state feedback and noise-assisted feedback individually.

The next main work is to design $\kappa\left(\rho_{t}\right)$ and prove the exponential convergence, which are the main contribution of this paper and will be presented in Section III. 


\section{COMBINED FEEDBACK CONTROL}

For the two-level quantum systems, there are two eigenstates, i.e., $\left\{\rho_{g}, \rho_{e}\right\}$, where $\rho_{g}=\left[\begin{array}{ll}1 & 0 \\ 0 & 0\end{array}\right]$ and $\rho_{e}=$ $\left[\begin{array}{ll}0 & 0 \\ 0 & 1\end{array}\right]$. The target eigenstate $\rho_{f}$ is set as $\rho_{g}$ in this section, the exponential stabilization of $\rho_{e}$ follows the same lines.

In order to obtain $\kappa\left(\rho_{t}\right)$, we divide the state space $S$ into two subspaces, denoted as $\Phi_{1}$ and $\Phi_{2}$, and the convergence rate under state feedback is faster that that under noise-assisted feedback if $\rho_{t} \in \Phi_{1}$, while the state convergence under noiseassisted feedback is faster that that under state feedback if $\rho_{t} \in \Phi_{2}=S \backslash \Phi_{1}$. As a result, $\kappa\left(\rho_{t}\right)$ can be designed as

$$
\kappa\left(\rho_{t}\right)=\left\{\begin{array}{l}
1, \text { if } \rho_{t} \in \Phi_{1} \\
0, \text { if } \rho_{t} \in \Phi_{2}
\end{array}\right.
$$

based on the idea of the combined feedback strategy. From (8), the key of obtaining $\kappa\left(\rho_{t}\right)$ is the partition of state space $S$, i.e. determining $\Phi_{1}$ and $\Phi_{2}$.

In [27], two continuous noise-assisted feedback are proposed, based on which we design two combined feedback strategies in this section, respectively.

\section{A. Combination of state feedback and linear noise-assisted feedback}

The continuous linear noise-assisted feedback control is designed as [27]

$$
u_{t}^{n f} d t=\alpha V\left(\rho_{t}\right) d B_{t}
$$

where, the constant $\alpha>0$ and

$$
V\left(\rho_{t}\right)=\sqrt{1-\operatorname{tr}\left(\rho_{t} \rho_{f}\right)}
$$

The state feedback control proposed in [19] is

$$
u_{t}^{s f} d t=\mu V^{\beta}\left(\rho_{t}\right) d t-\gamma \operatorname{tr}\left(\mathrm{i}\left[\sigma_{y}, \rho_{t}\right] \rho_{f}\right) d t
$$

where, $\gamma \geq 0, \beta \geq 1$ and $0<\mu<\frac{4 \eta \Gamma \nu^{2}}{(1-\nu)^{\frac{\beta-1}{2}}}$ with $\nu \in(0,1)$. From (5), (9) and (11), we can obtain a combined feedback control

$$
\begin{aligned}
u_{t}^{c f} d t= & \kappa\left(\rho_{t}\right) \mu V^{\beta}\left(\rho_{t}\right) d t-\kappa\left(\rho_{t}\right) \gamma \operatorname{tr}\left(\mathrm{i}\left[\sigma_{y}, \rho_{t}\right] \rho_{f}\right) d t \\
& +\left(1-\kappa\left(\rho_{t}\right)\right) \alpha V\left(\rho_{t}\right) d B_{t}
\end{aligned}
$$

i.e., $f\left(\rho_{t}\right)=\mu V^{\beta}\left(\rho_{t}\right) d t-\gamma \operatorname{tr}\left(\mathrm{i}\left[\sigma_{y}, \rho_{t}\right] \rho_{f}\right)$ and $g\left(\rho_{t}\right)=$ $\alpha V\left(\rho_{t}\right)$ in (5).

Based on (12), one of the main results of this paper is given in Theorem 1.

Theorem 1. For the quantum SME (7) under the combined feedback strategy (12) with the parameter

$$
\kappa\left(\rho_{t}\right)=\left\{\begin{array}{l}
1, \text { if } \rho_{t} \in \Phi_{1} \\
0, \text { if } \rho_{t} \in \Phi_{2}
\end{array}\right.
$$

with $\Phi_{1}=\left\{\rho \in S: \mu \operatorname{tr}\left(\mathrm{i}\left[\sigma_{y}, \rho\right] \rho_{f}\right) V^{\beta-2}(\rho)+\right.$ $\left(\frac{\alpha^{2}}{4}-\gamma\right) \frac{\operatorname{tr}^{2}\left(\mathrm{i}\left[\sigma_{y}, \rho\right] \rho_{f}\right)}{V^{2}(\rho)}<\alpha^{2}\left(1-2 V^{2}(\rho)\right)$ and

$\left.\nu<\operatorname{tr}\left(\rho \rho_{f}\right) \leq 1\right\}$ and $\Phi_{2}=S \backslash \Phi_{1}$, the Lyapunov function $V\left(\rho_{t}\right)=\sqrt{1-\operatorname{tr}\left(\rho_{t} \rho_{f}\right)}$ satisfies $\mathbb{E}\left[V\left(\rho_{t}\right)\right]<e^{-r t} V\left(\rho_{0}\right)$ for all $t \geq 0$, and the target eigenstate $\rho_{f}$ is globally exponentially stable with the convergence rate $r$ that is not more than $\min \left\{2 \eta \Gamma \nu^{2}-\mu(1-\nu)^{\frac{\beta-1}{2}}, \frac{1}{2} \eta \Gamma\right\}$. In particular, the state convergence under the combined feedback control (12) is faster than that under the noise-assisted feedback (9) and the state feedback control (11) individually.

Proof. When $\kappa\left(\rho_{t}\right)=1$, (7) becomes

$$
\begin{aligned}
d \rho_{t}= & \Gamma\left(\sigma_{z} \rho_{t} \sigma_{z}-\rho_{t}\right) d t-\mathrm{i} f\left(\rho_{t}\right)\left[\sigma_{y}, \rho_{t}\right] d t \\
& +\sqrt{\eta \Gamma}\left(\sigma_{z} \rho_{t}+\rho_{t} \sigma_{z}-2 \operatorname{tr}\left(\rho_{t} \sigma_{z}\right) \rho_{t}\right) d W_{t} \\
= & \Gamma\left(\sigma_{z} \rho_{t} \sigma_{z}-\rho_{t}\right) d t-\mathrm{i}\left(\mu \mathrm{V}^{\beta}\left(\rho_{\mathrm{t}}\right) \mathrm{dt}\right. \\
& \left.-\gamma \operatorname{tr}\left(\mathrm{i}\left[\sigma_{y}, \rho_{t}\right] \rho_{f}\right)\right)\left[\sigma_{y}, \rho_{t}\right] d t+\sqrt{\eta \Gamma}\left(\sigma_{z} \rho_{t}+\rho_{t} \sigma_{z}\right. \\
& \left.-2 \operatorname{tr}\left(\rho_{t} \sigma_{z}\right) \rho_{t}\right) d W_{t}
\end{aligned}
$$

and the infinitesimal generator $\mathcal{L}$ associated with (14) acts on $V$ is given by

$$
\begin{aligned}
\mathcal{L} V\left(\rho_{t}\right)= & \frac{\mu V^{\beta}\left(\rho_{t}\right)-\gamma \operatorname{tr}\left(\mathrm{i}\left[\sigma_{y}, \rho_{t}\right] \rho_{f}\right)}{2} \frac{\operatorname{tr}\left(\mathrm{i}\left[\sigma_{y}, \rho_{t}\right] \rho_{f}\right)}{V\left(\rho_{t}\right)} \\
& -2 \eta \Gamma \operatorname{tr}^{2}\left(\rho_{t} \rho_{f}\right) V\left(\rho_{t}\right) \\
= & \frac{\mu}{2} \operatorname{tr}\left(\mathrm{i}\left[\sigma_{y}, \rho_{t}\right] \rho_{f}\right) V^{\beta-1}\left(\rho_{t}\right)-\frac{\gamma \operatorname{tr}^{2}\left(\mathrm{i}\left[\sigma_{y}, \rho_{t}\right] \rho_{f}\right)}{2 V\left(\rho_{t}\right)} \\
& -2 \eta \Gamma \operatorname{tr}^{2}\left(\rho_{t} \rho_{f}\right) V\left(\rho_{t}\right) \\
= & -\frac{1}{2}\left(4 \eta \Gamma \operatorname{tr}^{2}\left(\rho_{t} \rho_{f}\right)+\frac{\gamma \operatorname{tr}^{2}\left(\mathrm{i}\left[\sigma_{y}, \rho_{t}\right] \rho_{f}\right)}{V^{2}\left(\rho_{t}\right)}\right. \\
& \left.-\mu \operatorname{tr}\left(\mathrm{i}\left[\sigma_{y}, \rho_{t}\right] \rho_{f}\right) V^{\beta-2}\left(\rho_{t}\right)\right) V\left(\rho_{t}\right)
\end{aligned}
$$

based on that the infinitesimal generator $\mathcal{L}$ associated with $d \rho_{t}=F\left(\rho_{t}\right) d t+G\left(\rho_{t}\right) d W_{t}$ acts on $V\left(\rho_{t}\right)$ in the following way,

$$
\mathcal{L} V\left(\rho_{t}\right)=\frac{\partial V\left(\rho_{t}\right)}{\partial \rho_{t}} F\left(\rho_{t}\right)+\frac{1}{2} \frac{\partial^{2} V\left(\rho_{t}\right)}{\partial \rho_{t}^{2}}\left(G\left(\rho_{t}\right), G\left(\rho_{t}\right)\right),
$$

where the Hessian is thought as a quadratic form.

$$
\text { When } \kappa\left(\rho_{t}\right)=0 \text {, (7) becomes }
$$

$$
\begin{aligned}
d \rho_{t}= & \Gamma\left(\sigma_{z} \rho_{t} \sigma_{z}-\rho_{t}\right) d t+g\left(\rho_{t}\right)^{2}\left(\sigma_{y} \rho_{t} \sigma_{y}-\rho_{t}\right) d t \\
& +\sqrt{\eta \Gamma}\left(\sigma_{z} \rho_{t}+\rho_{t} \sigma_{z}-2 \operatorname{tr}\left(\rho_{t} \sigma_{z}\right) \rho_{t}\right) d W_{t} \\
& -\mathrm{i} g\left(\rho_{t}\right)\left[\sigma_{y}, \rho_{t}\right] d B_{t} \\
= & \Gamma\left(\sigma_{z} \rho_{t} \sigma_{z}-\rho_{t}\right) d t+\alpha^{2} V^{2}\left(\rho_{t}\right)\left(\sigma_{y} \rho_{t} \sigma_{y}-\rho_{t}\right) d t \\
& +\sqrt{\eta \Gamma}\left(\sigma_{z} \rho_{t}+\rho_{t} \sigma_{z}-2 \operatorname{tr}\left(\rho_{t} \sigma_{z}\right) \rho_{t}\right) d W_{t} \\
& -\mathrm{i} \alpha V\left(\rho_{t}\right)\left[\sigma_{y}, \rho_{t}\right] d B_{t}
\end{aligned}
$$

and the infinitesimal generator $\mathcal{L}$ associated with (17) acts on 
$V$ is given by

$$
\begin{aligned}
\mathcal{L} V\left(\rho_{t}\right)= & -\frac{\alpha^{2} V^{2}\left(\rho_{t}\right)\left(\operatorname{tr}\left(\rho_{t} \rho_{e}\right)-\operatorname{tr}\left(\rho_{t} \rho_{f}\right)\right)}{2 \sqrt{1-\operatorname{tr}\left(\rho_{t} \rho_{f}\right)}} \\
& -\frac{\eta \Gamma \operatorname{tr}^{2}\left(\rho_{t} \rho_{f}\right)\left(1-\operatorname{tr}\left(\rho_{t} \sigma_{z}\right)\right)^{2}}{2\left(1-\operatorname{tr}\left(\rho_{t} \rho_{f}\right)\right)^{\frac{3}{2}}} \\
& -\frac{\alpha^{2} V^{2}\left(\rho_{t}\right) \operatorname{tr}^{2}\left(\mathrm{i}\left[\sigma_{y}, \rho_{t}\right] \rho_{f}\right)}{8\left(1-\operatorname{tr}\left(\rho_{t} \rho_{f}\right)\right)^{\frac{3}{2}}} \\
= & -\frac{\alpha^{2}}{2} V\left(\rho_{t}\right)\left(\operatorname{tr}\left(\rho_{t} \rho_{e}\right)-\operatorname{tr}\left(\rho_{t} \rho_{f}\right)\right) \\
& -\frac{\eta \Gamma}{2 V\left(\rho_{t}\right)^{3}} \operatorname{tr}^{2}\left(\rho_{t} \rho_{f}\right)\left(1-\operatorname{tr}\left(\rho_{t} \sigma_{z}\right)\right)^{2} \\
& -\frac{\alpha^{2} \operatorname{tr}^{2}\left(\mathrm{i}\left[\sigma_{y}, \rho_{t}\right] \rho_{f}\right)}{8 V\left(\rho_{t}\right)} \\
= & -\frac{1}{2}\left(\alpha^{2}\left(\operatorname{tr}^{2}\left(\rho_{t} \rho_{e}\right)-\operatorname{tr}\left(\rho_{t} \rho_{f}\right)\right)\right. \\
& +\frac{\eta \Gamma}{V\left(\rho_{t}\right)^{3}} \operatorname{tr}^{2}\left(\rho_{t} \rho_{f}\right)\left(1-\operatorname{tr}\left(\rho_{t} \sigma_{z}\right)\right)^{2} \\
& \left.+\frac{\alpha^{2} \operatorname{tr}^{2}\left(\mathrm{i}\left[\sigma_{y}, \rho_{t}\right] \rho_{f}\right)}{4 V^{2}\left(\rho_{t}\right)}\right) V\left(\rho_{t}\right)
\end{aligned}
$$

Because $\operatorname{tr}\left(\rho_{t}\right)=\operatorname{tr}\left(\rho_{t} \rho_{f}\right)+\operatorname{tr}\left(\rho_{t} \rho_{e}\right)=1$ and $\operatorname{tr}\left(\rho_{t} \sigma_{z}\right)=$ $\operatorname{tr}\left(\rho_{t} \rho_{f}\right)-\operatorname{tr}\left(\rho_{t} \rho_{e}\right)$,

$$
\begin{aligned}
1-\operatorname{tr}\left(\rho_{t} \sigma_{z}\right) & =1-\operatorname{tr}\left(\rho_{t} \rho_{f}\right)+\operatorname{tr}\left(\rho_{t} \rho_{e}\right) \\
& =2\left(1-\operatorname{tr}\left(\rho_{t} \rho_{f}\right)\right)
\end{aligned}
$$

from which

$$
\operatorname{tr}\left(\rho_{t} \rho_{e}\right)-\operatorname{tr}\left(\rho_{t} \rho_{f}\right)=2 V^{2}\left(\rho_{t}\right)-1
$$

Place (19) and (20) into (18), (18) becomes

$$
\begin{aligned}
\mathcal{L} V\left(\rho_{t}\right)= & -\frac{1}{2}\left(4 \eta \Gamma \operatorname{tr}^{2}\left(\rho_{t} \rho_{f}\right)+\frac{\alpha^{2}\left(\rho_{t}\right) \operatorname{tr}^{2}\left(\mathrm{i}\left[\sigma_{y}, \rho_{t}\right] \rho_{f}\right)}{4 V^{2}\left(\rho_{t}\right)}\right. \\
& \left.-\alpha^{2}\left(1-2 V^{2}\left(\rho_{t}\right)\right)\right) V\left(\rho_{t}\right)
\end{aligned}
$$

Compare (15) with (21), if $\rho_{t} \in \Phi_{1}$, the state convergence under state feedback is faster than that under noise-assisted feedback, while the state convergence under noise-assisted feedback is faster if $\rho_{t} \in \Phi_{2}$. From (13), the combined feedback strategy (12) use state feedback (11) in $\Phi_{1}$ and use noise-assisted feedback (9) in $\Phi_{2}$, such that the state convergence under the combined feedback (12) is faster than that under noise-assisted feedback (9) in $\Phi_{1}$, and is faster than that under state feedback (11) in $\Phi_{2}$, which means the state convergence under (12) is faster than that under (9) and (11) individually for the entire state space.

On the other hand, according to Theorem 4.3 in [19] and Theorem 1 in [27], under state feedback (11)

$$
\mathcal{L} V\left(\rho_{t}\right) \leq-\left(2 \eta \Gamma \nu^{2}-\mu(1-\nu)^{\frac{\beta-1}{2}}\right) V\left(\rho_{t}\right)
$$

while under noise-assisted feedback (9)

$$
\mathcal{L} V\left(\rho_{t}\right) \leq-\frac{1}{2} \eta \Gamma V\left(\rho_{t}\right)
$$

thus, under the combined feedback (12)

$$
\mathcal{L} V\left(\rho_{t}\right) \leq-\min \left\{2 \eta \Gamma \nu^{2}-\mu(1-\nu)^{\frac{\beta-1}{2}}, \frac{1}{2} \eta \Gamma\right\} V\left(\rho_{t}\right)
$$

Because $V\left(\rho_{t}\right)=0$ if and only if $\rho_{t}=\rho_{f}$, according to Theorem 3 in [25], one can obtained from (24) that

$\mathbb{E}\left[V\left(\rho_{t}\right)\right] \leq V\left(\rho_{0}\right) e^{-\min \left\{2 \eta \Gamma \nu^{2}-\mu(1-\nu)^{\frac{\beta-1}{2}}, \frac{1}{2} \eta \Gamma\right\} t} \leq V\left(\rho_{0}\right) e^{-r t}$

which means that $\rho_{f}$ is globally exponentially stable, and the convergence rate $r$ is not more than $\min \left\{2 \eta \Gamma \nu^{2}-\mu(1-\nu)^{\frac{\beta-1}{2}}, \frac{1}{2} \eta \Gamma\right\}$. The proof is finished.

Remark 1. It should be noted that the comparison conclusion of real state convergence rate is not consistent with that of the minimum convergence rate, thus we divide the state space $S$ by using real state convergence rate instead of the minimum convergence rate in the proof of Theorem 1.

B. Combination of state feedback and exponential noiseassisted feedback

The continuous exponential noise-assisted feedback is design as [27]

$$
\begin{aligned}
u_{t}^{n f} d t & =\lambda\left(e^{V\left(\rho_{t}\right)}-1\right) V\left(\rho_{t}\right) d B_{t} \\
& =\Lambda\left(\rho_{t}\right) d B_{t}
\end{aligned}
$$

where, $\Lambda\left(\rho_{t}\right)=\lambda\left(e^{V\left(\rho_{t}\right)}-1\right)$, from which we can obtain the other combined feedback control

$$
\begin{aligned}
u_{t}^{c f} d t= & \kappa\left(\rho_{t}\right) \mu V^{\beta}\left(\rho_{t}\right) d t-\kappa\left(\rho_{t}\right) \gamma \operatorname{tr}\left(\mathrm{i}\left[\sigma_{y}, \rho_{t}\right] \rho_{f}\right) d t \\
& +\left(1-\kappa\left(\rho_{t}\right)\right) \Lambda\left(\rho_{t}\right) V\left(\rho_{t}\right) d B_{t}
\end{aligned}
$$

i.e., $f\left(\rho_{t}\right)=\mu V^{\beta}\left(\rho_{t}\right) d t-\gamma \operatorname{tr}\left(\mathrm{i}\left[\sigma_{y}, \rho_{t}\right] \rho_{f}\right)$ and $g\left(\rho_{t}\right)=$ $\Lambda\left(\rho_{t}\right) V\left(\rho_{t}\right)$ in (5).

Based on (27), the other main results of this paper is given in Theorem 2.

Theorem 2. For the quantum SME (7) under the combined feedback strategy (27) with the parameters

$$
\kappa\left(\rho_{t}\right)=\left\{\begin{array}{l}
1, \text { if } \rho_{t} \in \Phi_{1} \\
0, \text { if } \rho_{t} \in \Phi_{2}
\end{array}\right.
$$

with $\Phi_{1}=\left\{\rho \in S: \mu \operatorname{tr}\left(\mathrm{i}\left[\sigma_{y}, \rho\right] \rho_{f}\right) V^{\beta-2}(\rho)+\right.$ $\left(\frac{\Lambda^{2}\left(\rho_{t}\right)}{4}-\gamma\right) \frac{\operatorname{tr}^{2}\left(\mathrm{i}\left[\sigma_{y}, \rho\right] \rho_{f}\right)}{V^{2}(\rho)}<\Lambda^{2}\left(\rho_{t}\right)\left(1-2 V^{2}(\rho)\right)$

and $\left.\nu<\operatorname{tr}\left(\rho \rho_{f}\right) \leq 1\right\}$ and $\Phi_{2}=S \backslash \Phi_{1}$, the Lyapunov function $V\left(\rho_{t}\right)=\sqrt{1-\operatorname{tr}\left(\rho_{t} \rho_{f}\right)}$ satisfies $\mathbb{E}\left[V\left(\rho_{t}\right)\right]<$ $e^{-r t} V\left(\rho_{0}\right)$ for all $t \geq 0$, and the target eigenstate $\rho_{f}$ is globally exponentially stable with the convergence rate $r$ that is not more than $\min \left\{2 \eta \Gamma \nu^{2}-\mu(1-\nu)^{\frac{\beta-1}{2}}, \frac{1}{2} \eta \Gamma\right\}$. In particular, the state convergence under the combined feedback control (27) is faster than that under the noise-assisted feedback (26) and the state feedback control (11) individually. 
Proof. When $\kappa\left(\rho_{t}\right)=0$, (17) becomes

$$
\begin{aligned}
d \rho_{t}= & \Gamma\left(\sigma_{z} \rho_{t} \sigma_{z}-\rho_{t}\right) d t+\Lambda^{2}\left(\rho_{t}\right) V^{2}\left(\rho_{t}\right)\left(\sigma_{y} \rho_{t} \sigma_{y}-\rho_{t}\right) d t \\
& +\sqrt{\eta \Gamma}\left(\sigma_{z} \rho_{t}+\rho_{t} \sigma_{z}-2 \operatorname{tr}\left(\rho_{t} \sigma_{z}\right) \rho_{t}\right) d W_{t} \\
& -\mathrm{i} \Lambda\left(\rho_{t}\right) V\left(\rho_{t}\right)\left[\sigma_{y}, \rho_{t}\right] d B_{t}
\end{aligned}
$$

and the infinitesimal generator $\mathcal{L}$ associated with (29) acts on $V$ is given by

$$
\begin{aligned}
\mathcal{L} V\left(\rho_{t}\right)= & -\frac{\Lambda^{2}\left(\rho_{t}\right) V^{2}\left(\rho_{t}\right)\left(\operatorname{tr}\left(\rho_{t} \rho_{e}\right)-\operatorname{tr}\left(\rho_{t} \rho_{f}\right)\right)}{2 \sqrt{1-\operatorname{tr}\left(\rho_{t} \rho_{f}\right)}} \\
& -\frac{\eta \Gamma \operatorname{tr}^{2}\left(\rho_{t} \rho_{f}\right)\left(1-\operatorname{tr}\left(\rho_{t} \sigma_{z}\right)\right)^{2}}{2\left(1-\operatorname{tr}\left(\rho_{t} \rho_{f}\right)\right)^{\frac{3}{2}}} \\
& -\frac{\Lambda^{2}\left(\rho_{t}\right) V^{2}\left(\rho_{t}\right) \operatorname{tr}^{2}\left(\mathrm{i}\left[\sigma_{y}, \rho_{t}\right] \rho_{f}\right)}{8\left(1-\operatorname{tr}\left(\rho_{t} \rho_{f}\right)\right)^{\frac{3}{2}}} \\
= & -\frac{\Lambda^{2}\left(\rho_{t}\right)}{2} V\left(\rho_{t}\right)\left(\operatorname{tr}\left(\rho_{t} \rho_{e}\right)-\operatorname{tr}\left(\rho_{t} \rho_{f}\right)\right) \\
& -\frac{\eta \Gamma}{2 V\left(\rho_{t}\right)^{3}} \operatorname{tr}^{2}\left(\rho_{t} \rho_{f}\right)\left(1-\operatorname{tr}\left(\rho_{t} \sigma_{z}\right)\right)^{2} \\
& -\frac{\Lambda^{2}\left(\rho_{t}\right) \operatorname{tr}^{2}\left(\mathrm{i}\left[\sigma_{y}, \rho_{t}\right] \rho_{f}\right)}{8 V\left(\rho_{t}\right)} \\
= & -\frac{1}{2}\left(\Lambda^{2}\left(\rho_{t}\right)\left(\operatorname{tr}\left(\rho_{t} \rho_{e}\right)-\operatorname{tr}\left(\rho_{t} \rho_{f}\right)\right)\right. \\
& +\frac{\eta \Gamma}{V\left(\rho_{t}\right)^{3}} \operatorname{tr}^{2}\left(\rho_{t} \rho_{f}\right)\left(1-\operatorname{tr}\left(\rho_{t} \sigma_{z}\right)\right)^{2} \\
& \left.+\frac{\Lambda^{2}\left(\rho_{t}\right) \operatorname{tr}^{2}\left(\mathrm{i}\left[\sigma_{y}, \rho_{t}\right] \rho_{f}\right)}{4 V^{2}\left(\rho_{t}\right)}\right) V\left(\rho_{t}\right)
\end{aligned}
$$

Place (19) and (20) into (30), (30) becomes

$$
\begin{aligned}
\mathcal{L} V\left(\rho_{t}\right)= & -\frac{1}{2}\left(4 \eta \Gamma \operatorname{tr}^{2}\left(\rho_{t} \rho_{f}\right)+\frac{\Lambda^{2}\left(\rho_{t}\right) \operatorname{tr}^{2}\left(\mathrm{i}\left[\sigma_{y}, \rho_{t}\right] \rho_{f}\right)}{4 V^{2}\left(\rho_{t}\right)}\right. \\
& \left.-\Lambda^{2}\left(\rho_{t}\right)\left(1-2 V^{2}\left(\rho_{t}\right)\right)\right) V\left(\rho_{t}\right)
\end{aligned}
$$

According to the similar discussion in the proof of Theorem 1 , the state convergence under the combined feedback (27) is faster than that under the noise-assisted feedback (26) and the state feedback (11) individually for the entire state space.

On the other hand, according to Theorem 2 in [27], under noise-assisted feedback (26)

$$
\mathcal{L} V\left(\rho_{t}\right) \leq-\frac{1}{2} \eta \Gamma V\left(\rho_{t}\right)
$$

thus, under the combined feedback (27)

$$
\mathcal{L} V\left(\rho_{t}\right) \leq-\min \left\{2 \eta \Gamma \nu^{2}-\mu(1-\nu)^{\frac{\beta-1}{2}}, \frac{1}{2} \eta \Gamma\right\} V\left(\rho_{t}\right)
$$

which means

$\mathbb{E}\left[V\left(\rho_{t}\right)\right] \leq V\left(\rho_{0}\right) e^{-\min \left\{2 \eta \Gamma \nu^{2}-\mu(1-\nu)^{\frac{\beta-1}{2}}, \frac{1}{2} \eta \Gamma\right\} t} \leq V\left(\rho_{0}\right) e^{-r t}$

based on Theorem 3 in [25]. Thus, $\rho_{f}$ is globally exponentially stable, and the convergence rate $r$ is not more than $\min \left\{2 \eta \Gamma \nu^{2}-\mu(1-\nu)^{\frac{\beta-1}{2}}, \frac{1}{2} \eta \Gamma\right\}$. The proof is finished.

\section{NUMERICAL SIMULATIONS}

In this section, we use the combined feedback controls (12) and (27) to exponentially stabilize the target eigenstate $\rho_{e}$ with initial state $\rho_{0}=\rho_{g}$, respectively, and compare the two combined feedback controls.

A. Exponential stabilization of $\rho_{e}$ by using the combined feedback control (12)

Let the physical parameters $\eta=0.5, \Gamma=0.25$, and the control parameters $\alpha=0.5, \mu=0.5, \gamma=0.05, \beta=8$. Under these parameters, $\min \left\{2 \eta \Gamma \nu^{2}-\mu(1-\nu)^{\frac{\beta-1}{2}}, \frac{1}{2} \eta \Gamma\right\}=\frac{1}{2} \eta \Gamma$. The results of 100 experiments with $\nu=0.8$ are shown in Fig. 1, from which one can see that the quantum state $\rho_{t}$ converge to the target eigenstate $\rho_{e}$ from the initial state $\rho_{g}$ exponentially, which means the combined feedback control (12) is available.
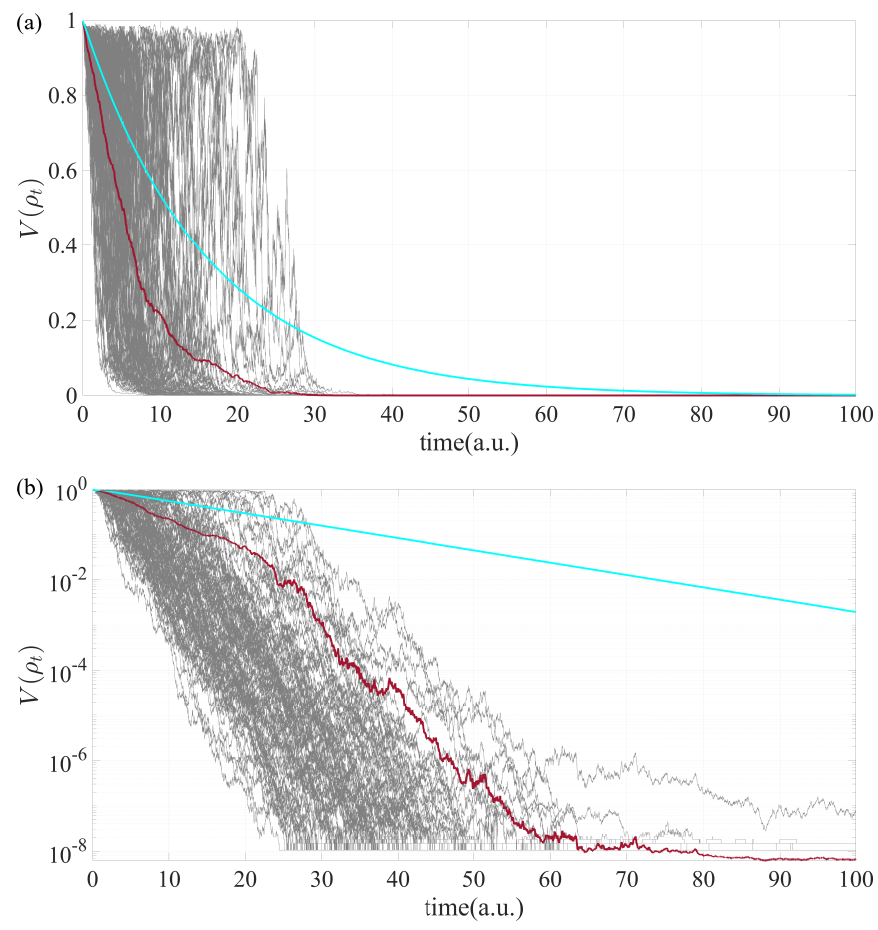

Fig. 1: Exponential stabilization of the target eigenstate $\rho_{e}$ for the quantum system (7) by using the combined feedback control (12) starting at $\rho_{g}$ with $\alpha=0.5, \mu=0.5, \gamma=0.05$ and $\beta=8$. (a) The curves of $V\left(\rho_{t}\right)$ in 100 arbitrary sample trajectories, and the red curve represents the mean value of 100 arbitrary sample trajectories, while the cyan curve represents the exponential reference with exponent $-\frac{1}{2} \eta \Gamma$; (b) The semi$\log$ version of (a).

In order to present the superiority of the combined feedback, we also use the continuous linear noise-assisted feedback (9) and state feedback (11) to exponentially stabilize $\rho_{e}$ from initial state $\rho_{0}=\rho_{g}$, respectively. The results of 100 experiments with $\nu=0.8$ are shown in Fig. 2, from which one can see the state convergence is faster under the combined feedback (12) than that under the noise-assisted feedback (9) and state feedback (11), which reflects the superiority of the 
combined feedback control. Moreover, it can be observed from the Fig. 2 that the state convergence is faster under the noiseassisted feedback (9) than that under state feedback (11) in the initial stage of the control process, which is consistent with the theoretical results as described in the proof of Theorem 1. However, when the state closes to the target eigenstate, the state convergence is faster under the state feedback (11) than that under noise-assisted feedback (9). The reason is that the exiguous noise hinders the attraction of target state though it can make the system state "shake it away" from the bad equilibria to fight against the attraction of bad equilibria. Thus, the more reasonable control strategy is to use noise-assisted feedback when the state closes to bad equilibria and to use state feedback when the state closes to the target state, which is the idea of the combined feedback strategy proposed in this paper. A sample trajectory is presented in Fig. 3.
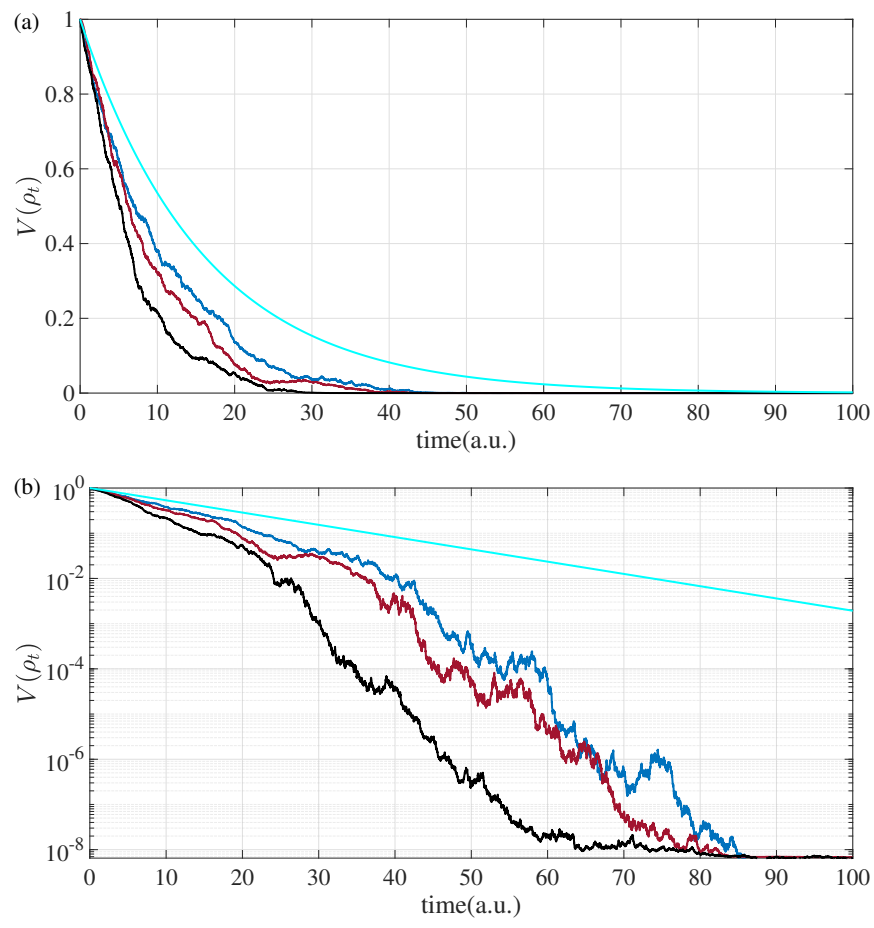

Fig. 2: Exponential stabilization of the target eigenstate $\rho_{e}$ for the quantum system (7) by using the continuous linear noiseassisted feedback (9) and state feedback (11) starting at $\rho_{g}$ with $\alpha=0.5, \mu=0.5, \gamma=0.05$ and $\beta=8$. (a) The blue, red and black curves represent the mean value of 100 arbitrary sample trajectories under the noise-assisted feedback (9), state feedback (11) and the combined feedback (12), respectively, while the cyan curve represents the exponential reference with exponent $-\frac{1}{2} \eta \Gamma$; (b) The semi-log version of (a).

We also study the influence of the control parameters $\alpha$ on the convergence rate. Let $\alpha=0.4,0.5$ and 0.6 , respectively, the results of 100 experiments are shown in Fig. 4, from which one can see that the larger the $\alpha$, the faster the state convergence, which means the larger $\alpha$ helps to improve the state convergence rate.
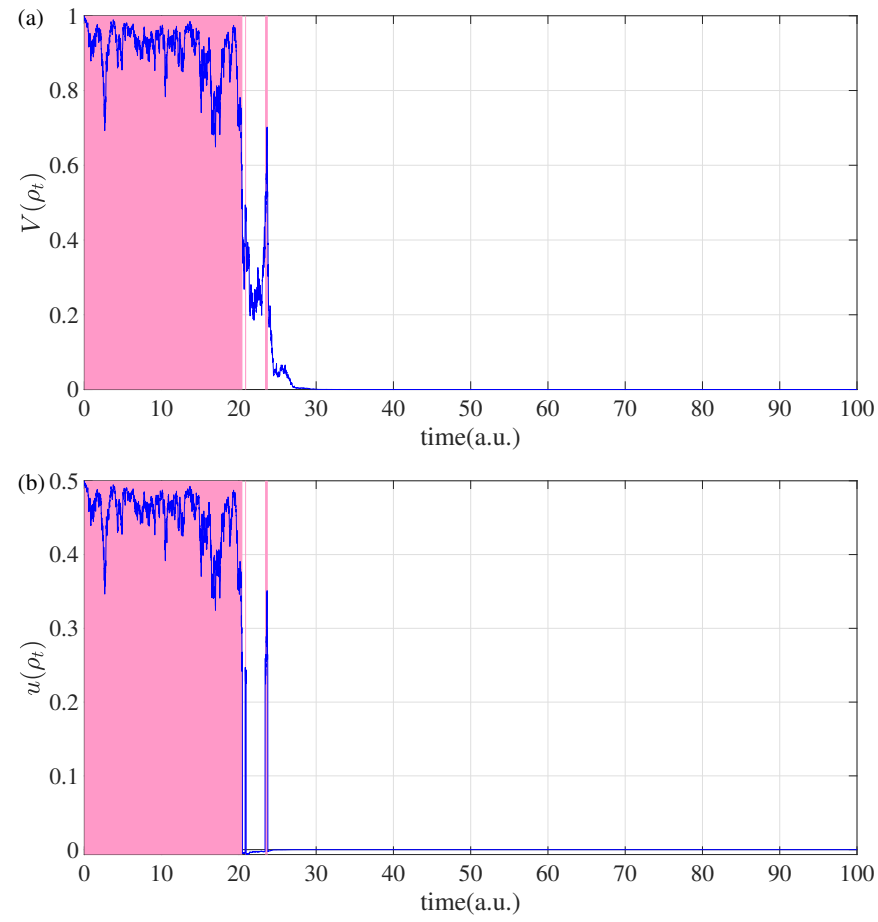

Fig. 3: A sample trajectory of exponential stabilization of the target eigenstate $\rho_{e}$ for the quantum system (7) by using the combined feedback (12) starting at $\rho_{g}$ with $\alpha=0.5, \mu=$ $0.5, \gamma=0.05$ and $\beta=8$. (a) The curve of $V\left(\rho_{t}\right)$ under the combined feedback (12); (b) The curve of the combined feedback control $u\left(\rho_{t}\right)$. The pink background represents the time domain of noise-assisted feedback (9), while the white background represents the time domain of state feedback (11).

\section{B. Exponential stabilization of $\rho_{e}$ by using the combined feedback control (27)}

For the combined feedback control (27), let $\lambda=\frac{0.5}{e^{V\left(\rho_{g}\right)}-1}$, and the other parameters are the same as that in subsection IV-A, the results of 100 experiments are shown in Fig. 5. One can see from Fig. 5 that the quantum state $\rho_{t}$ converge to the target eigenstate $\rho_{e}$ from the initial state $\rho_{g}$ exponentially, which means the effectiveness of the combined feedback control (27) is verified.

The results of comparing the continuous exponential noiseassisted feedback (26) and state feedback (11) with the combined feedback (27) are shown in Fig. 6, from which one can see that the state convergence is faster under the combined feedback (27) than that under noise-assisted feedback (26) and state feedback (11), which presents the superiority of the combined feedback (27). Besides, we compare the two combined feedback controls (12) and (27), whose experiment results are shown in Fig. 7, from which one can see that the state convergence is faster under (12) than that under (27), which is consistent with the theoretical results as described in [27]. The influences of control parameters $\lambda, \mu, \gamma$ and $\beta$ on convergence rate can be found in [19], so we ignore the corresponding experiment results here. Therefore, in order to achieve faster state convergence, the continuous linear noise- 

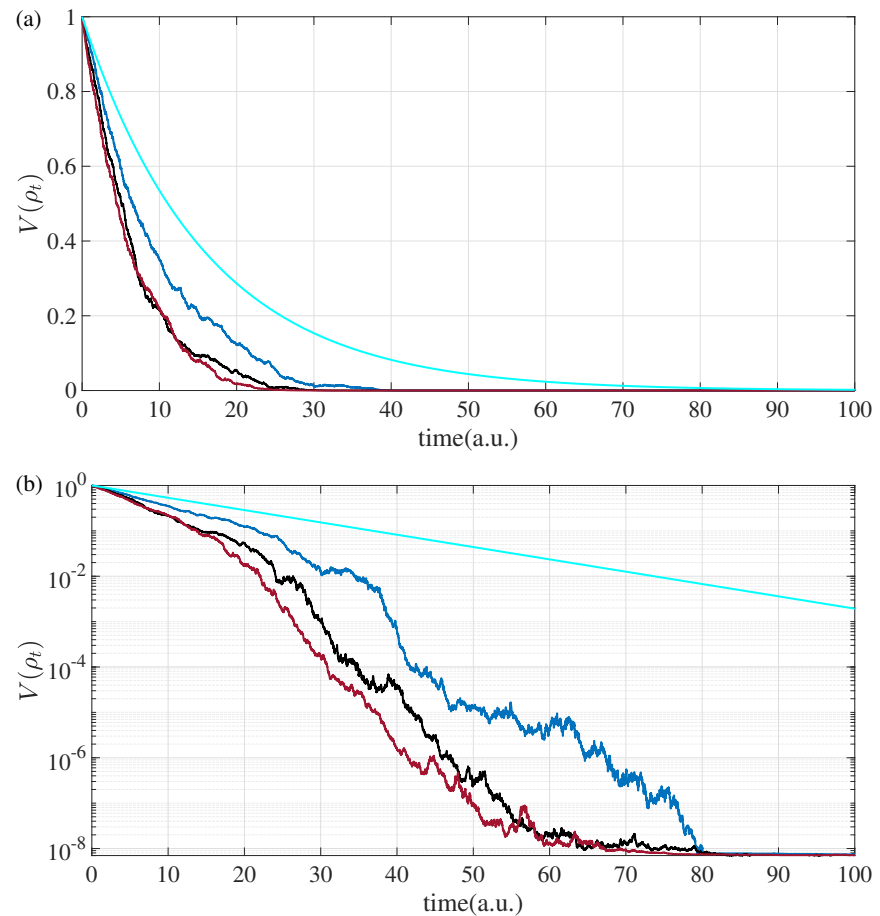

Fig. 4: Exponential stabilization of the target eigenstate $\rho_{e}$ for the quantum system (7) by using the combined feedback (12) starting at $\rho_{g}$ with $\mu=0.5, \gamma=0.05, \beta=8$ and $\alpha=0.4,0.5,0.6$, respectively. (a) The blue, black and red curves represent the mean value of 100 arbitrary sample trajectories for $\alpha=0.4,0.5,0.6$, respectively, while the cyan curve represents the exponential reference with exponent $-\frac{1}{2} \eta \Gamma$; (b) The semi-log version of (a).

assisted feedback should be used in the combined feedback strategy, in which larger $\alpha, \mu, \beta$ and $\gamma$ are recommended based on the results in Fig. 4 and the discussions in [19].

\section{CONCLUSIONS}

In this paper, we combine the state feedback and noiseassisted feedback to achieve the exponential stabilization of target eigenstate for two-level stochastic quantum systems, and the state convergence under the combined feedback is faster than that under the noise-assisted feedback and state feedback individually. Moreover, numerical simulations are used to verify the effectiveness and superiority of the combined feedback strategies. The further works can be considered as follows: (1) The robustness analysis of the combined feedback strategies when the physical parameters $\eta, \Gamma$ or the initial state $\rho_{0}$ are uncertain; (2) The exponential stabilization of $N$-level stochastic quantum systems, i.e. expanding the controlled systems to the more general stochastic quantum systems; (3) Exponential stabilization of time-delay stochastic quantum systems, specially the case of time-delay control, i.e. $u_{t}=u\left(\rho_{t-\tau}\right)$ with delay time $\tau$.

\section{REFERENCES}

[1] D. D'Alessandro and M. Dahleh, "Optimal control of two-level quantum systems," IEEE Transactions on Automatic Control, vol. 46, no. 6, pp. 866-876, Jun. 2001.
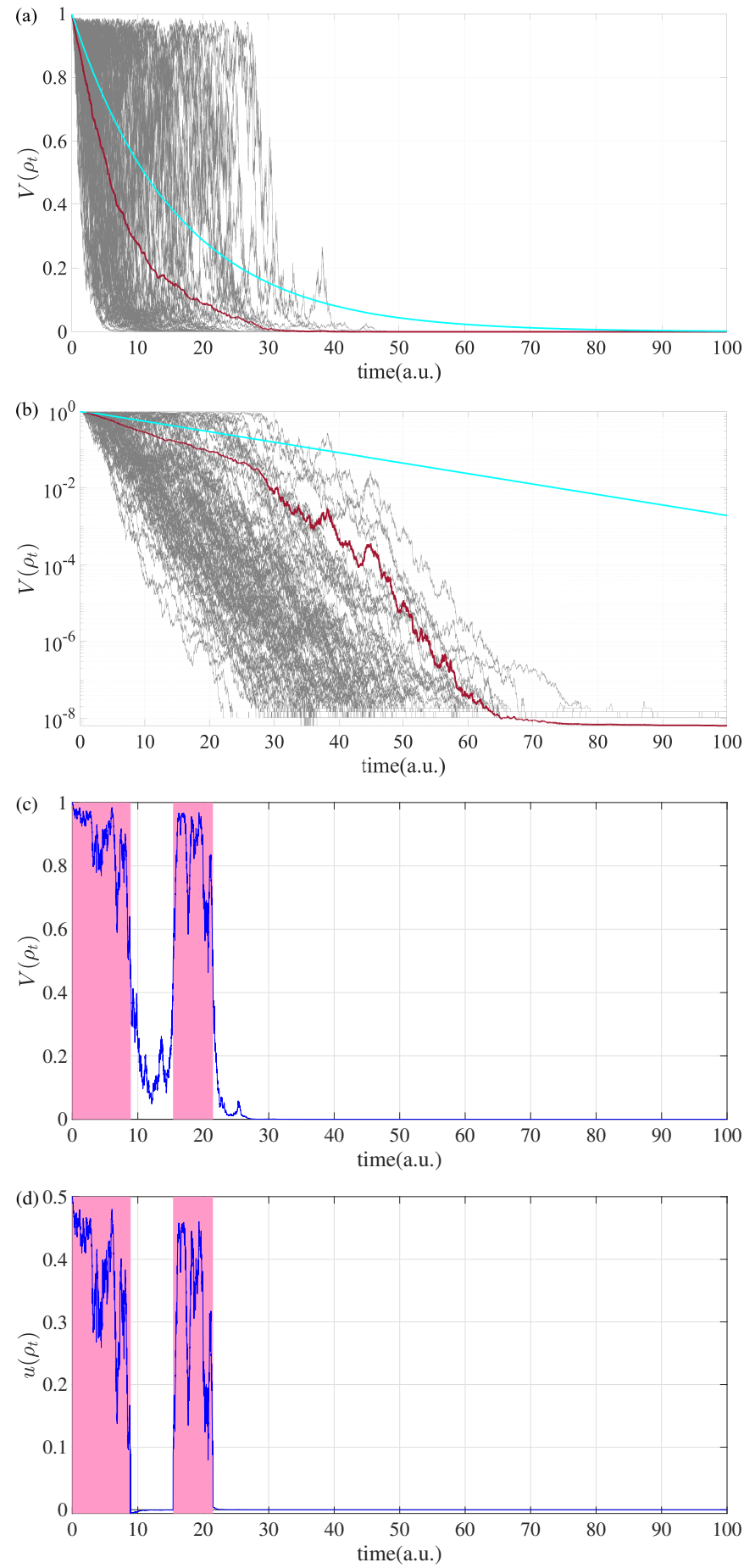

Fig. 5: Exponential stabilization of the target eigenstate $\rho_{e}$ for the quantum system (7) by using the combined feedback control (27) starting at $\rho_{g}$ with $\lambda=\frac{0.5}{e^{V\left(\rho_{g}\right)}-1}, \mu=0.5$, $\gamma=0.05$ and $\beta=8$. (a) The curves of $V\left(\rho_{t}\right)$ in 100 arbitrary sample trajectories, and the red curve represents the mean value of 100 arbitrary sample trajectories, while the cyan curve represents the exponential reference with exponent $-\frac{1}{2} \eta \Gamma$; (b) The semi-log version of (a); (c) The curve of $V\left(\rho_{t}\right)$ under the combined feedback control (27) in a sample trajectory; (d) The curve of the combined feedback control $u\left(\rho_{t}\right)$ in a sample trajectory. The pink background presents the time domain of noise-assisted feedback (26), while the white background represents the time domain of state feedback (11). 

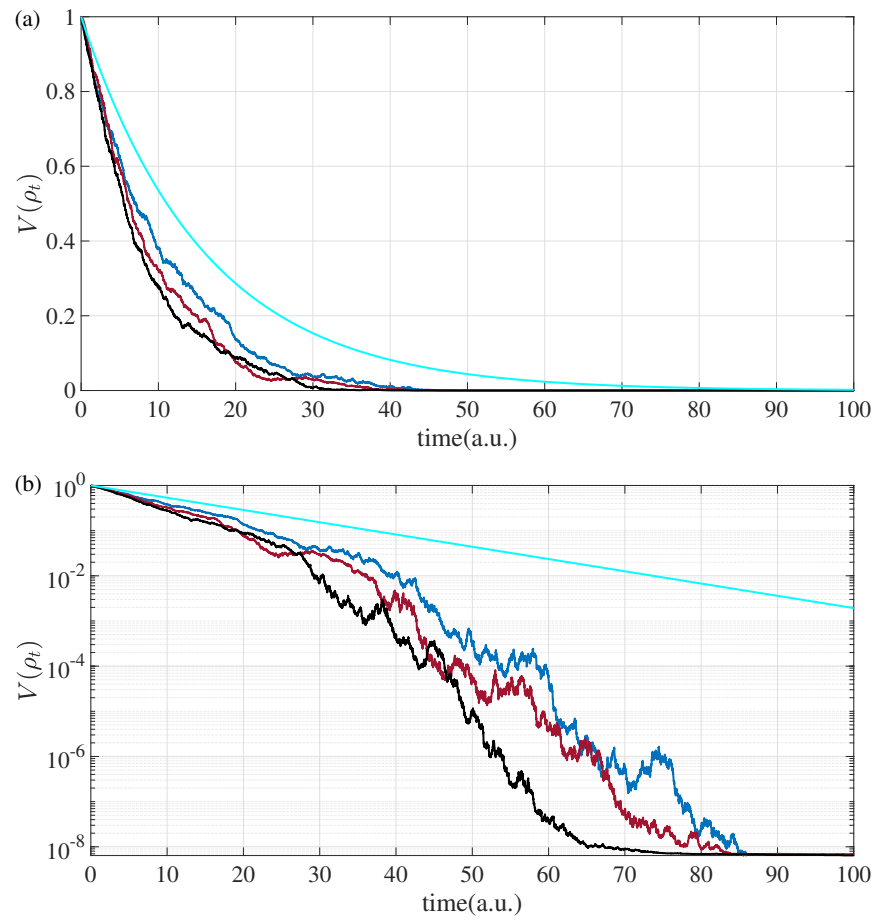

Fig. 6: Exponential stabilization of the target eigenstate $\rho_{e}$ for the quantum system (7) by using the continuous linear noiseassisted feedback (26) and state feedback (11) starting at $\rho_{g}$ with $\lambda=\frac{0.5}{e^{V\left(\rho_{g}\right)}-1}, \mu=0.5, \gamma=0.05$ and $\beta=8$. (a) The blue, red and black curves represent the mean value of 100 arbitrary sample trajectories under the noise-assisted feedback (26), state feedback (11) and the combined feedback (27), respectively, while the cyan curve represents the exponential reference with exponent $-\frac{1}{2} \eta \Gamma$; (b) The semi-log version of (a).

[2] H. Jirari, F. W. J. Hekking, and O. Buisson, "Optimal control of superconducting $N$-level quantum systems," EPL (Europhysics Letters), vol. 87, no. 2, p. 28004, Jul. 2009.

[3] D. Dong and I. R. Petersen, "Notes on sliding mode control of twolevel quantum systems," Automatica, vol. 48, no. 12, pp. 3089-3097, Dec. 2012.

[4] — , "Sliding mode control of two-level quantum systems," Automatica, vol. 48, no. 5, pp. 725-735, May 2012.

[5] A. I. Maalouf and I. R. Petersen, "Time-varying $H_{\infty}$ control for a class of linear quantum systems: A dynamic game approach," Automatica, vol. 48, no. 11, pp. 2908-2916, Nov. 2012.

[6] S. Kuang and S. Cong, "Lyapunov control methods of closed quantum systems," Automatica, vol. 44, no. 1, pp. 98-108, Jan. 2008.

[7] S. Kuang, D. Dong, and I. R. Petersen, "Rapid Lyapunov control of finite-dimensional quantum systems," Automatica, vol. 81, pp. 164-175, Jul. 2017.

[8] S. Zhao, H. Lin, J. Sun, and L. Zhou, "Implicit Lyapunov-based control strategy for closed quantum systems with dipole and polarizability coupling," International Journal of Robust and Nonlinear Control, vol. 27, no. 17, pp. 3886-3903, 2017.

[9] V. P. Belavkin, "On the theory of controlling observable quantum systems," Automation and Remote Control, vol. 44, no. 2, pp. 178-188, 1983.

[10] — "Quantum stochastic calculus and quantum nonlinear filtering," Journal of Multivariate Analysis, vol. 42, no. 2, pp. 171-201, Aug. 1992.

[11] L. Bouten, R. van Handel, and M. R. James, "A discrete invitation to quantum filtering and feedback control," SIAM Review, vol. 51, no. 2 , pp. 239-316, May 2009.

[12] R. van Handel, J. K. Stockton, and H. Mabuchi, "Feedback control
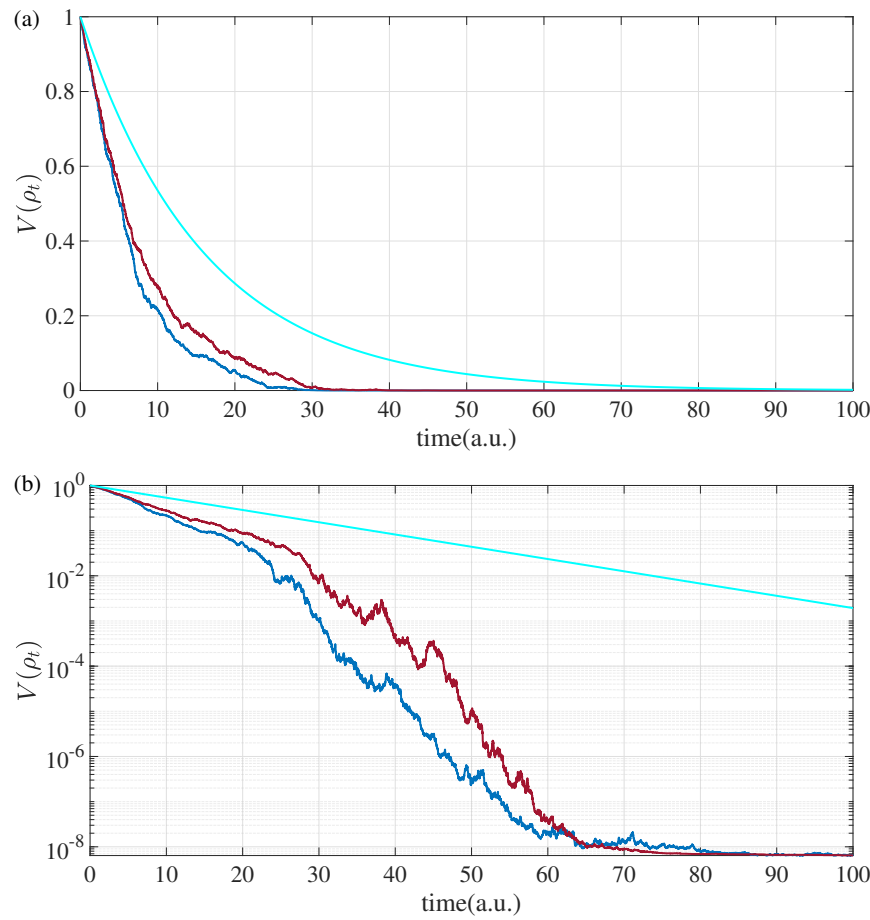

Fig. 7: Exponential stabilization of the target eigenstate $\rho_{e}$ for the quantum system (7) by using the combined feedback controls (12) and (27) starting at $\rho_{g}$ with $\alpha=0.5$, $\lambda=\frac{0.5}{e^{V\left(\rho_{g}\right)}-1}, \mu=0.5, \gamma=0.05$ and $\beta=8$. (a) The blue and red curves represent the mean value of 100 arbitrary sample trajectories under the combined feedback controls (12) and (27), respectively, while the cyan curve represents the exponential reference with exponent $-\frac{1}{2} \eta \Gamma$; (b) The semi-log version of (a).

of quantum state reduction," IEEE Transactions on Automatic Control, vol. 50, no. 6, pp. 768-780, Jun. 2005.

[13] M. Mirrahimi and R. van Handel, "Stabilizing feedback controls for quantum systems," SIAM Journal on Control and Optimization, vol. 46 , no. 2, pp. 445-467, Jan. 2007.

[14] S. Cong, J. Wen, S. Kuang, and F. Meng, "Global stabilization control of stochastic quantum systems," Science China Information Sciences, vol. 59, no. 11, p. 112502 , Oct. 2016.

[15] K. Tsumura, "Global stabilization of $N$-dimensional quantum spin systems via continuous feedback," in 2007 American Control Conference, Jul. 2007, pp. 2129-2134.

[16] — "Global stabilization at arbitrary eigenstates of $N$-dimensional quantum spin systems via continuous feedback," in 2008 American Control Conference, Jun. 2008, pp. 4148-4153.

[17] S. S. Ge, T. L. Vu, and C. C. Hang, "Non-smooth Lyapunov functionbased global stabilization for quantum filters," Automatica, vol. 48, no. 6 , pp. 1031-1044, Jun. 2012.

[18] T. L. Vu, S. S. Ge, and T. H. Lee, "Coupling Lyapunov functions approach for quantum control," in 2015 54th IEEE Conference on Decision and Control (CDC), Dec. 2015, pp. 7749-7754.

[19] W. Liang, N. H. Amini, and P. Mason, "On exponential stabilization of spin- $\frac{1}{2}$ systems," in 2018 IEEE Conference on Decision and Control (CDC), Dec. 2018, pp. 6602-6607.

[20] - "On exponential stabilization of $N$-level quantum angular momentum systems," SIAM Journal on Control and Optimization, vol. 57, no. 6, pp. 3939-3960, Jan. 2019.

[21] D. W. Stroock and S. R. S. Varadhan, "On the support of diffusion processes with applications to the strong maximum principle," in Proceedings of the Sixth Berkeley Symposium on Mathematical Statistics and Probability, Volume 3: Probability Theory. The Regents of the University of California, 1972. 
[22] X. Mao, "Stochastic versions of the LaSalle theorem," Journal of Differential Equations, vol. 153, no. 1, pp. 175-195, Mar. 1999.

[23] W. Liang, N. H. Amini, and P. Mason, "On the robustness of stabilizing feedbacks for quantum spin-1/2 systems," in 2020 59th IEEE Conference on Decision and Control (CDC), 2020, pp. 3842-3847.

[24] W. Liang, N. H. Amini, and P. Mason, "Robust feedback stabilization of $N$-level quantum spin systems," SIAM Journal on Control and Optimization, vol. 59, no. 1, pp. 669-692, 2021.

[25] G. Cardona, A. Sarlette, and P. Rouchon, "Exponential stabilization of quantum systems under continuous non-demolition measurements," Automatica, vol. 112, p. 108719, Feb. 2020.

[26] R. Khasminskii, Stochastic Stability of Differential Equations, 2nd ed. ser. Stochastic Modelling and Applied Probability. Berlin Heidelberg: Springer-Verlag, 2012.

[27] J. Wen, Y. Shi, J. Jia, and J. Zeng, "Exponential stabilization of twolevel quantum systems based on continuous noise-assisted feedback," to appear in Results in Physics, 2021.

[28] C. Pellegrini, "Existence, uniqueness and approximation of a stochastic schrödinger equation: The diffusive case," The Annals of Probability, vol. 36, no. 6, pp. 2332-2353, 2008.

[29] L. K. Thomsen, S. Mancini, and H. M. Wiseman, "Continuous quantum nondemolition feedback and unconditional atomic spin squeezing," Journal of Physics B: Atomic, Molecular and Optical Physics, vol. 35, no. 23, pp. 4937-4952, nov 2002.

[30] N. Yamamoto, K. Tsumura, and S. Hara, "Feedback control of quantum entanglement in a two-spin system," Automatica, vol. 43, no. 6, pp. 981 - 992, 2007

[31] J. Wang and H. M. Wiseman, "Feedback-stabilization of an arbitrary pure state of a two-level atom," Physical Review A, vol. 64, no. 6, p. 063810, Nov. 2001.

[32] F. Ticozzi and L. Viola, "Quantum Markovian subsystems: Invariance, attractivity, and control," IEEE Transactions on Automatic Control, vol. 53, no. 9, pp. 2048-2063, Oct. 2008.

[33] _ "Analysis and synthesis of attractive quantum Markovian dynamics," Automatica, vol. 45, no. 9, pp. 2002-2009, Sep. 2009.

[34] J. Wen, Y. Shi, and X. Lu, "Stabilizing a class of mixed states for stochastic quantum systems via switching control," Journal of the Franklin Institute, vol. 355, no. 5, pp. 2562-2582, Mar. 2018.

[35] J. Wen, Y. Shi, X. Pang, J. Jia, and J. Zeng, "Rapid stabilization of time delay stochastic quantum systems based on continuous measurement feedback," Journal of the Franklin Institute, vol. 357, no. 12, pp. 75157536, Aug. 2020. 